\title{
Research on the climate changes and the simulation of the runoff over the last 50 years, 1961-2010, in the Upper Tarim River basin of Southern Xinjiang, China
}

\author{
Hongyi $\mathrm{Li}^{1}$, ZiQiang $\mathrm{Ma}^{2}$, Leiding Ding ${ }^{3}$, Zhen $\mathrm{Gao}^{4}$, Jintao $\mathrm{Xu}^{5}$, Kang $\mathrm{He}^{6}$, Chunhui \\ Feng $^{7}$, and Jie Peng ${ }^{7}$ \\ ${ }^{1}$ Jiangxi University of Finance and Economics \\ ${ }^{2}$ Peking University \\ ${ }^{3}$ Guizhou University \\ ${ }^{4}$ Guangdong Research Institute of Water Resources and Hydropower \\ ${ }^{5}$ Zhejiang University \\ ${ }^{6}$ University of Connecticut \\ ${ }^{7}$ Tarim University
}

April 28, 2020

\begin{abstract}
The upper Tarim River basin is supporting about 50 million people by melting the glaciers and snow, which are highly vulnerable and sensitive to climate change. Therefore, assessing the relative effects of climate change on runoff of this region is essential not only for understanding the mechanism of hydrological response over the mountainous areas in Southern Xinjiang but also for local water resources management. This study quantitatively investigated the climate change in the mountainous area of the upper Tarim River basin, using the up-to-date 'ground-truth' precipitation and temperature data, the APHRODITE (1961$\left.2010,0.25^{\circ}\right)$ data; analyzed the potential connections between runoff data, observed at Alar station, and the key climatological variables; and discussed the regression models on simulating the runoff based on precipitation and temperature data. The main findings of this study are: (1) both annual precipitation and temperature generally increases at rates of $0.85 \mathrm{~mm} / \mathrm{year}$ and 0.25 /10a, respectively, while the runoff data measured at Alar station shows fluctuating decreasing trends; (2) there are significant spatial differences in the temporal trends of precipitation, for example, the larger increasing rates of precipitation occurs in the Karakoram Mountains, while the larger decreasing rates happens in northwestern of Kashgar County; (3) the decreasing trends of temperature mainly occurs in the Kashgar County and its surrounding areas in Summer; (4) seasonal correlations in trends of precipitation and temperature are more significant than those at monthly and annual scale; and (5) the regression model in simulating the runoff in the upper Tarim River basin based on Radial Basis Function (RBF) is better than that based on least-squares method, with the predictive values based on RBF models significantly better (Correlation coefficient, CC, $0.85)$ than those by least-squares models $\left(\mathrm{CC}^{\sim} 0.75\right)$. These findings will provide valuable information to inform environmental scientists and planners on the climate change issues in the upper Tarim River basin of Southern Xinjiang, China, under a semiarid-arid cl
\end{abstract}

\section{Hosted file}

1.Manuscript.pdf available at https://authorea.com/users/308848/articles/439895-research-onthe-climate-changes-and-the-simulation-of-the-runoff-over-the-last-50-years-1961-2010-inthe-upper-tarim-river-basin-of-southern-xinjiang-china 


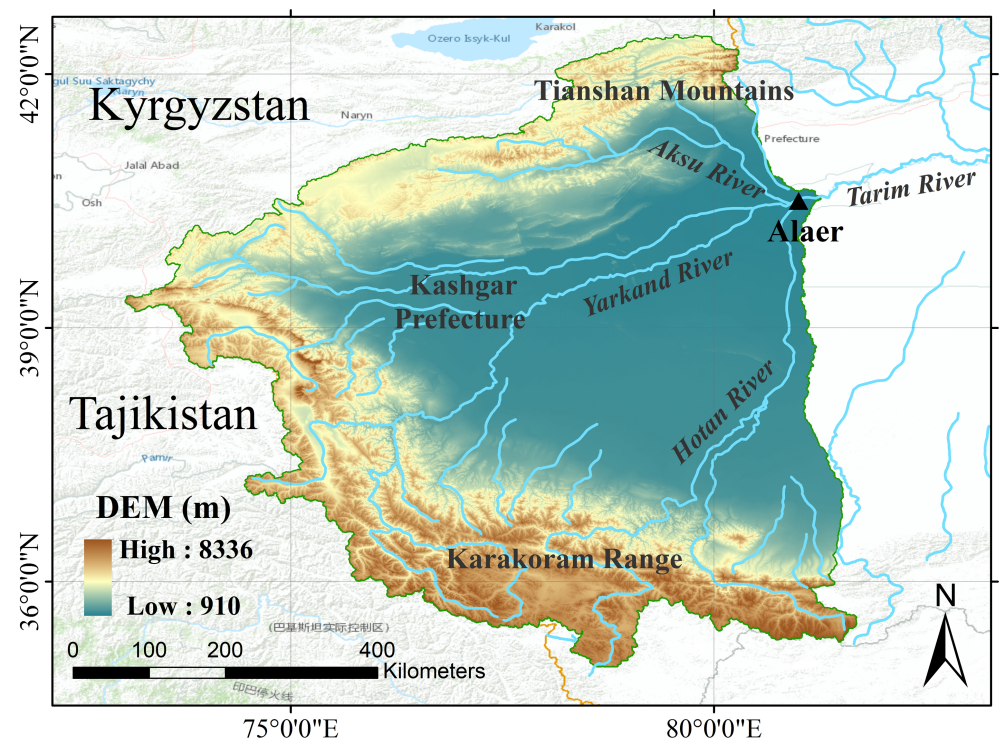

(a)

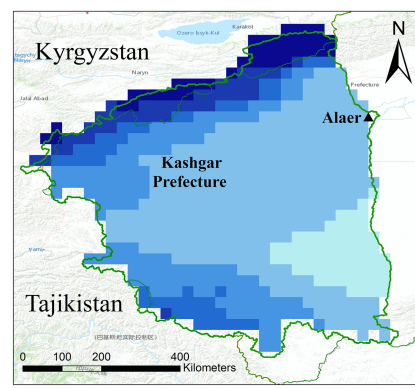

(b)

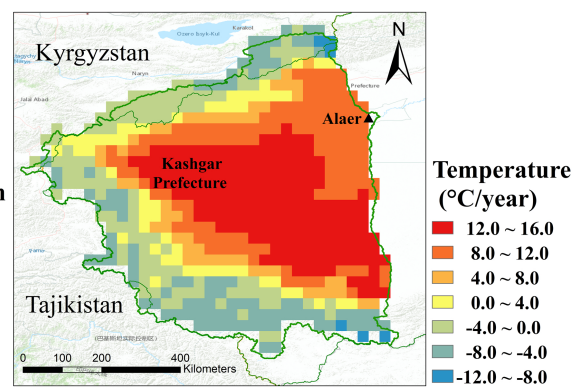

(c)

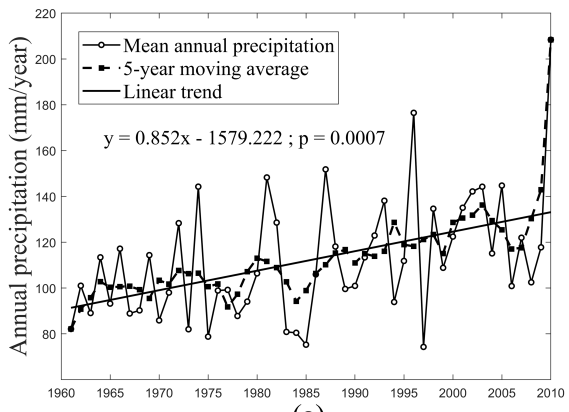

(a)

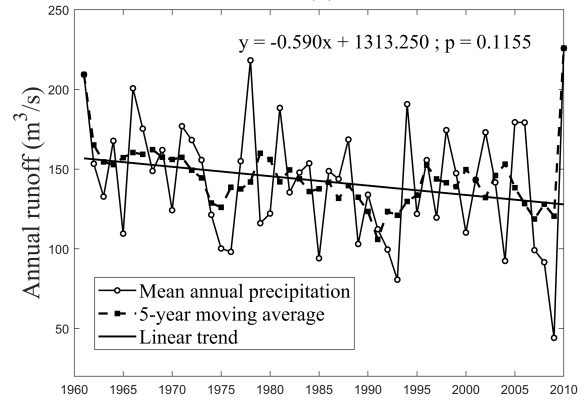

(c)

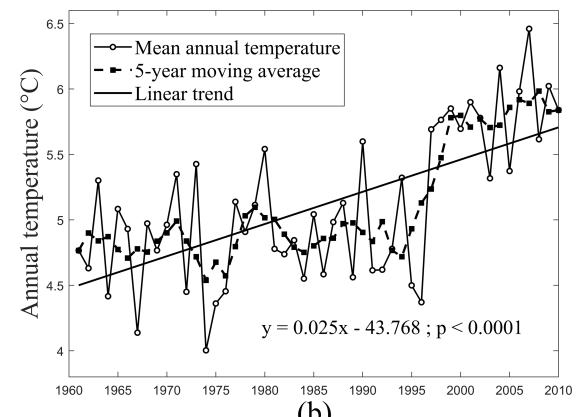

(b) 


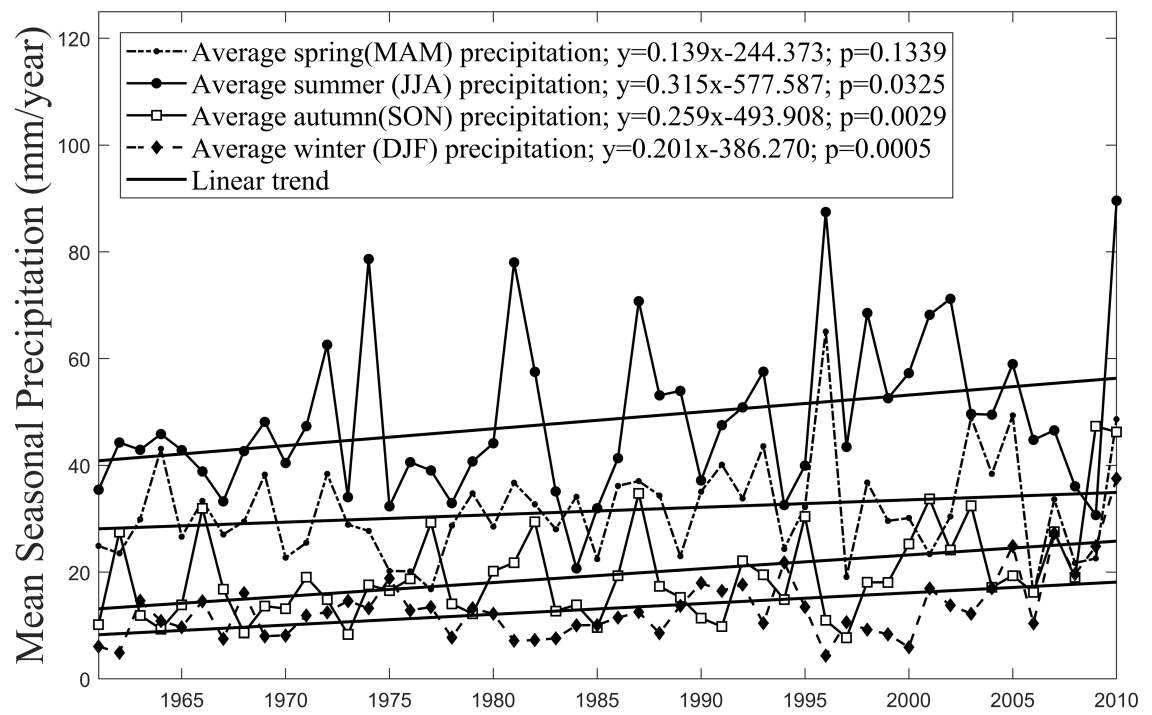

(a)

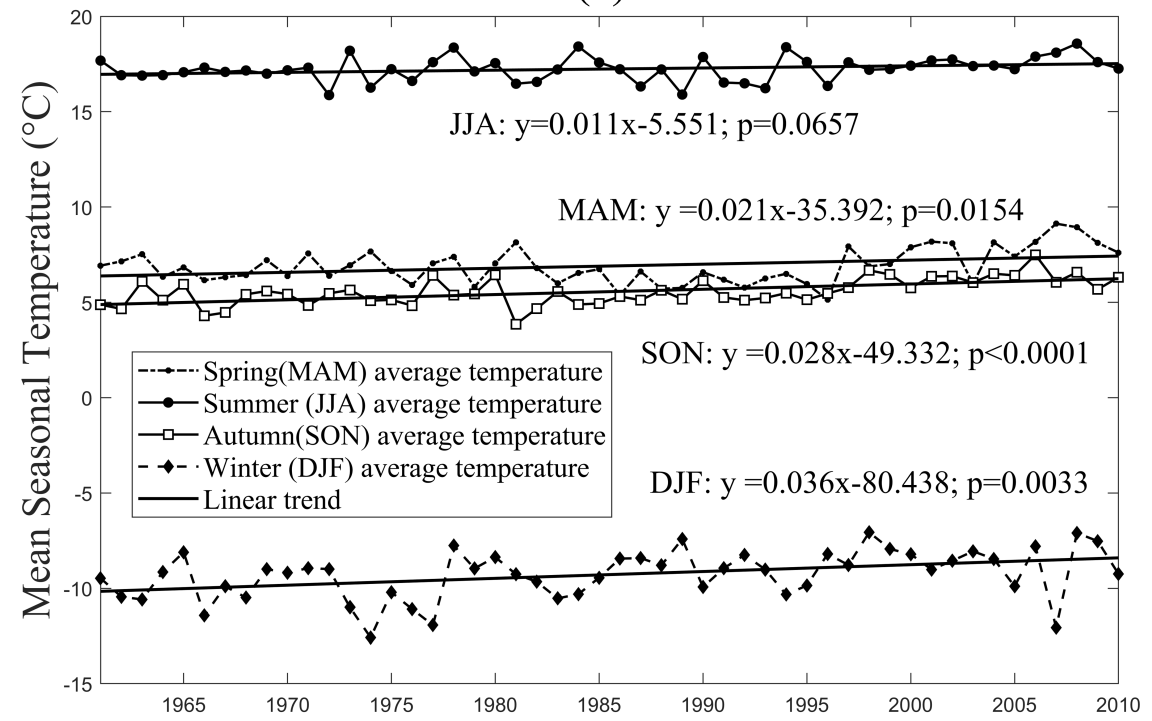

(b)

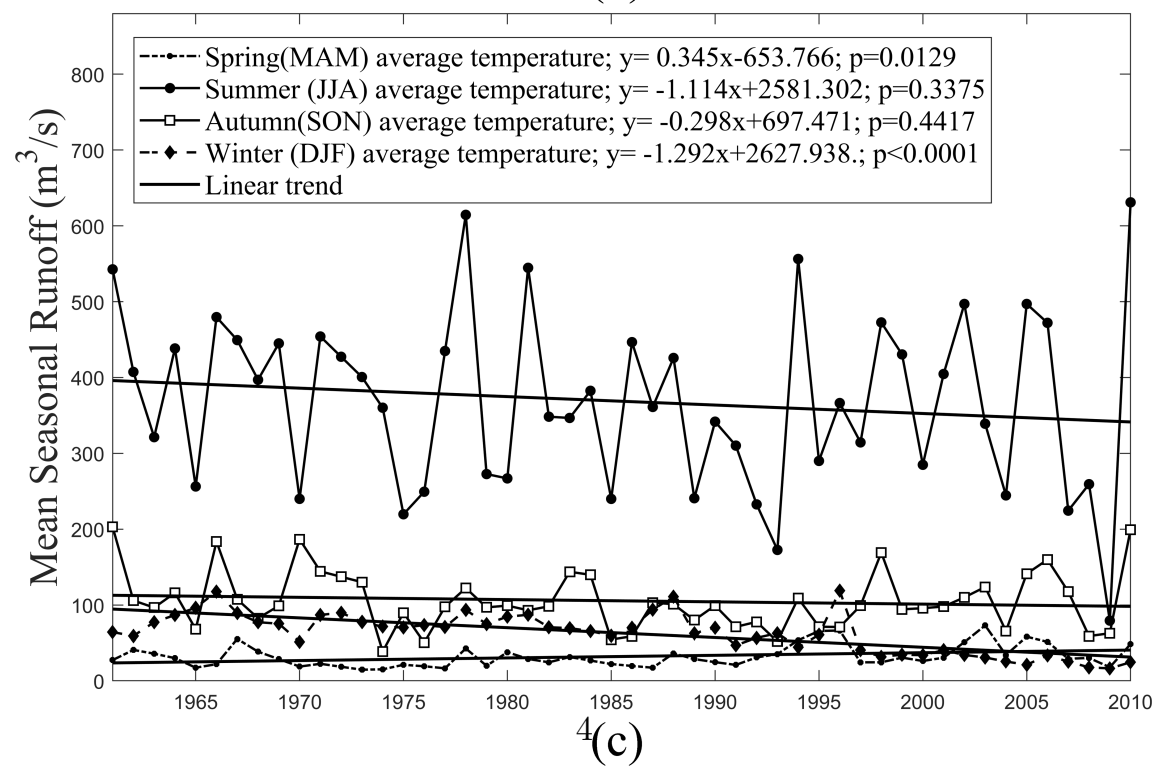



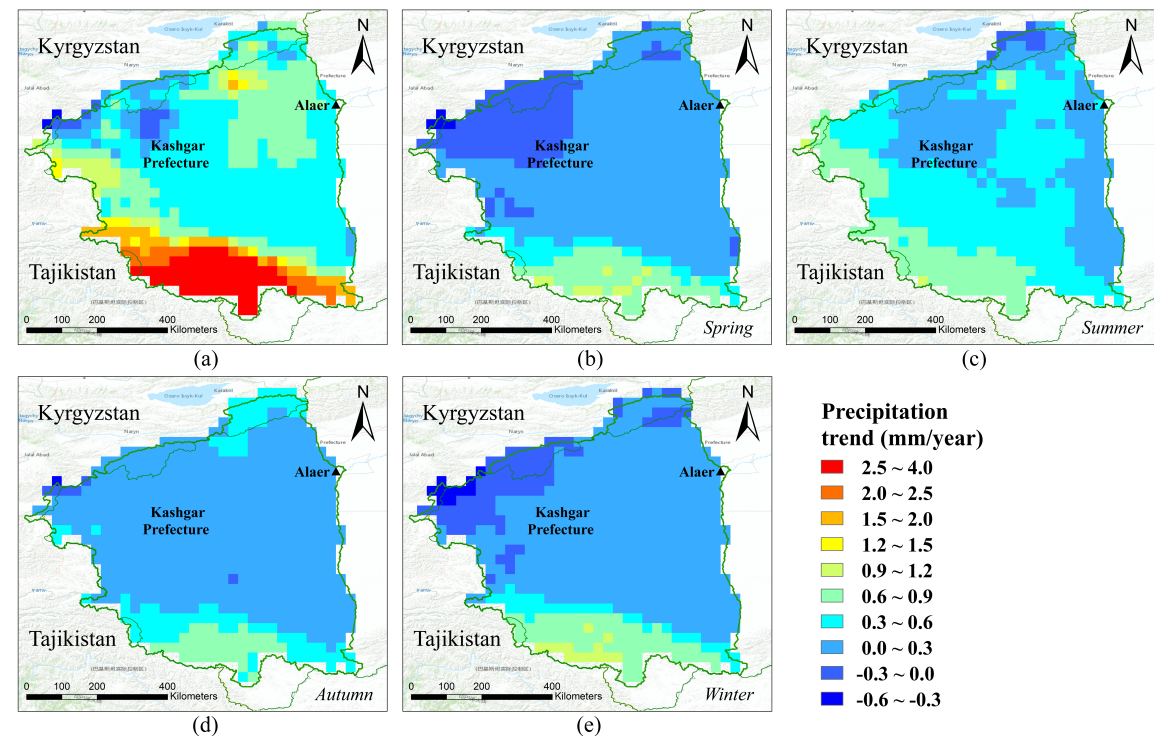

Precipitation

trend ( $\mathrm{mm} / \mathrm{year})$

- $2.5 \sim 4.0$

- $2.0 \sim 2.5$

$\square \quad 1.5 \sim 2.0$

$\square \quad 1.2 \sim 1.5$

$\square \quad 0.9 \sim 1.2$

$\square \quad 0.6 \sim 0.9$

$\square \quad 0.3 \sim 0.6$

$0.0 \sim 0.3$

- $-0.3 \sim 0.0$

(e)
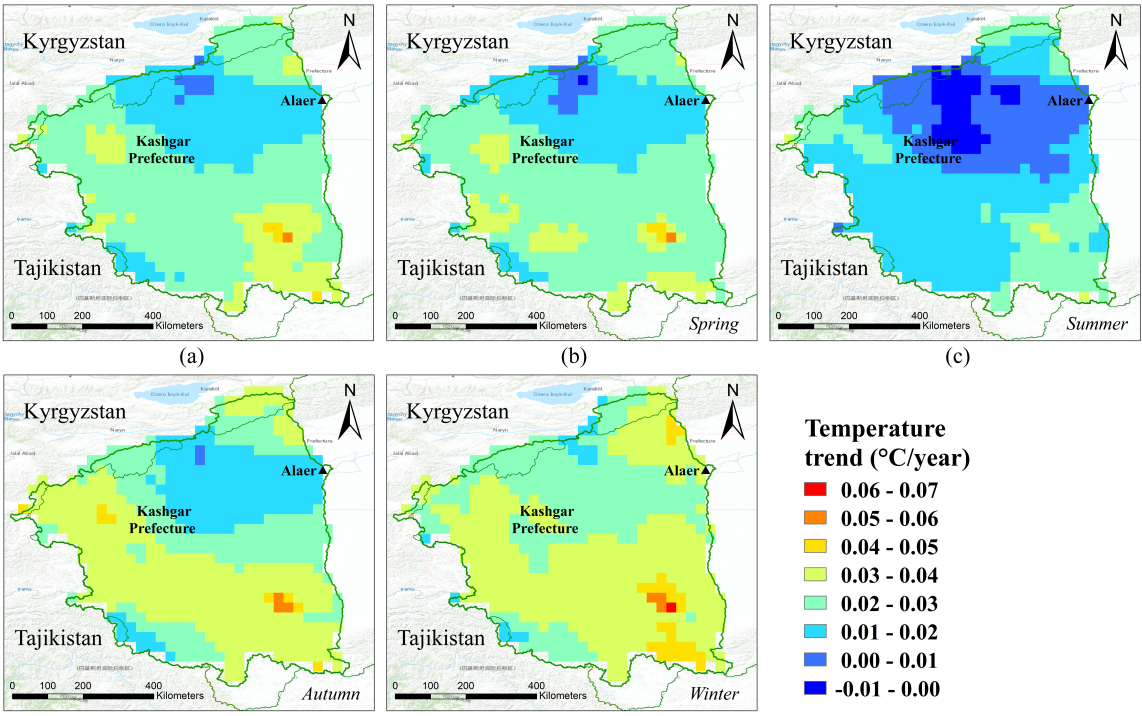

(b)

(c)

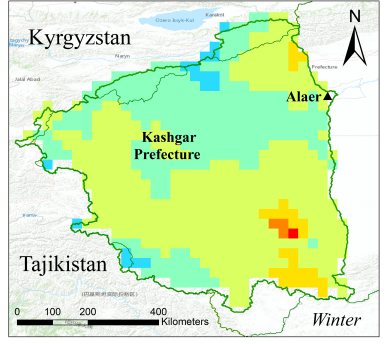

Temperature

trend $\left({ }^{\circ} \mathrm{C} /\right.$ year $)$

- 0.06 - 0.07

$0.05-0.06$

$\square 0.04-0.05$

$\square 0.03-0.04$

$\square .02-0.03$

$0.01-0.02$

$0.00-0.01$

- -0.01 - 0.00

(d)

(e) 


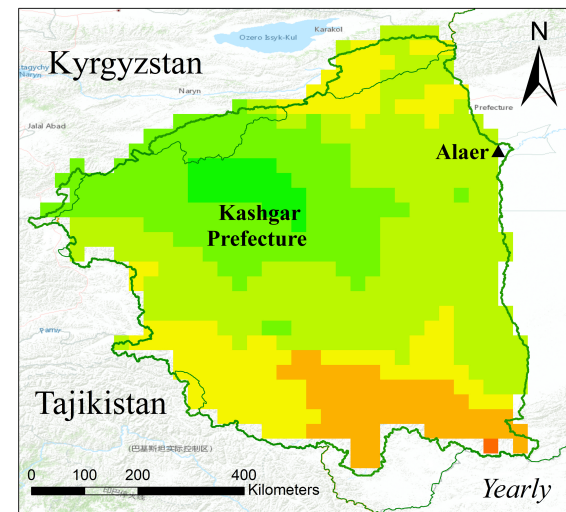

(a)

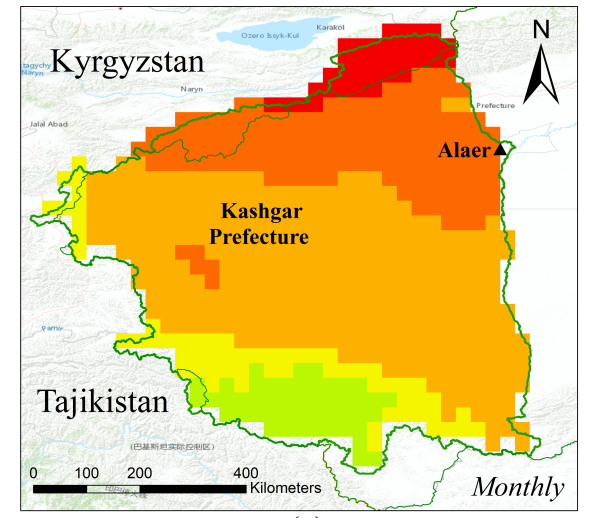

(c)

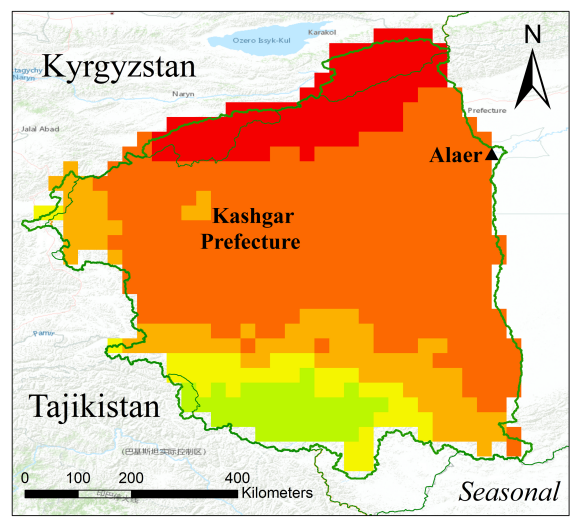

(b)

\section{Correlation} coefficient

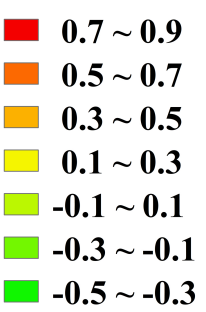

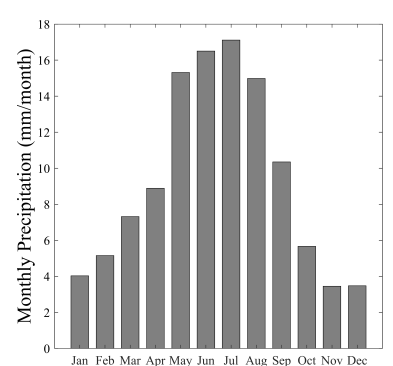

(a)

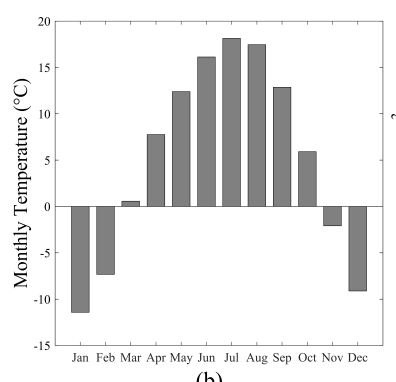

(b)

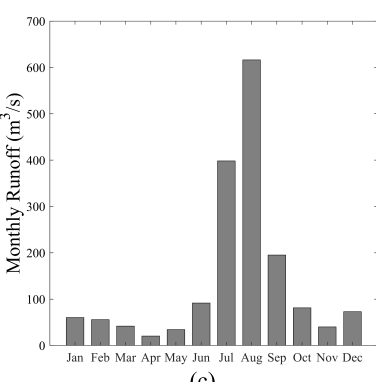

(c) 


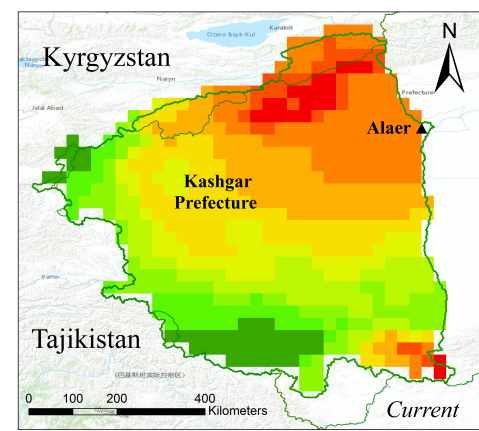

(a)

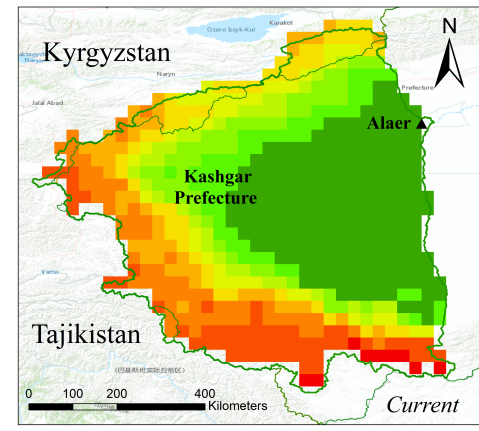

(c)

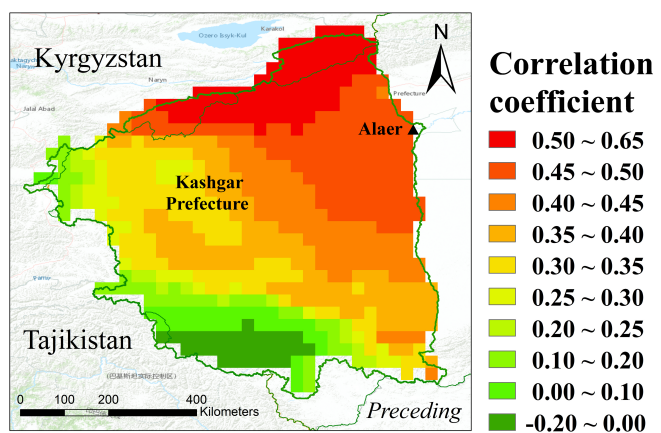

(b)

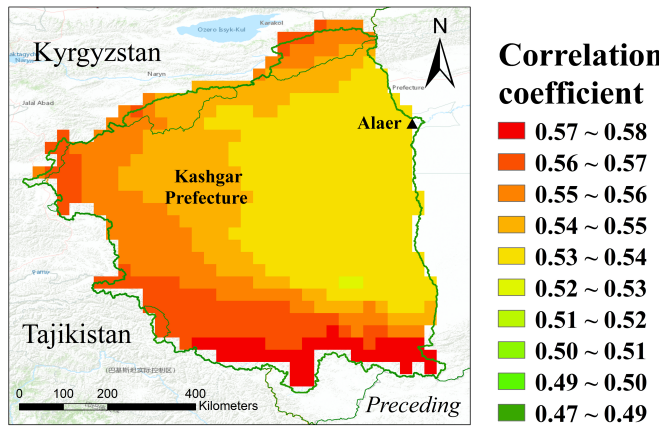

(d)

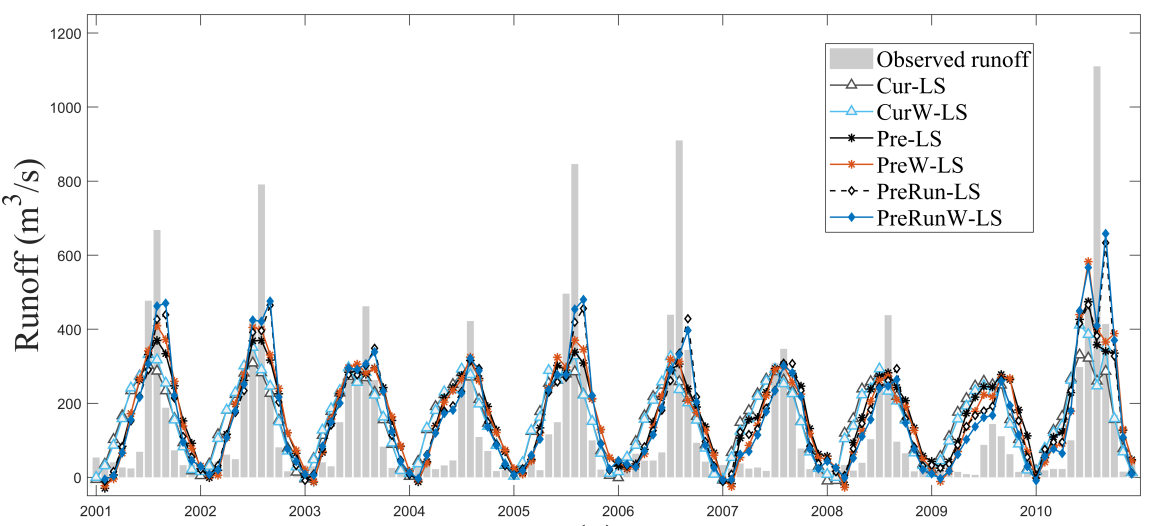

(a)

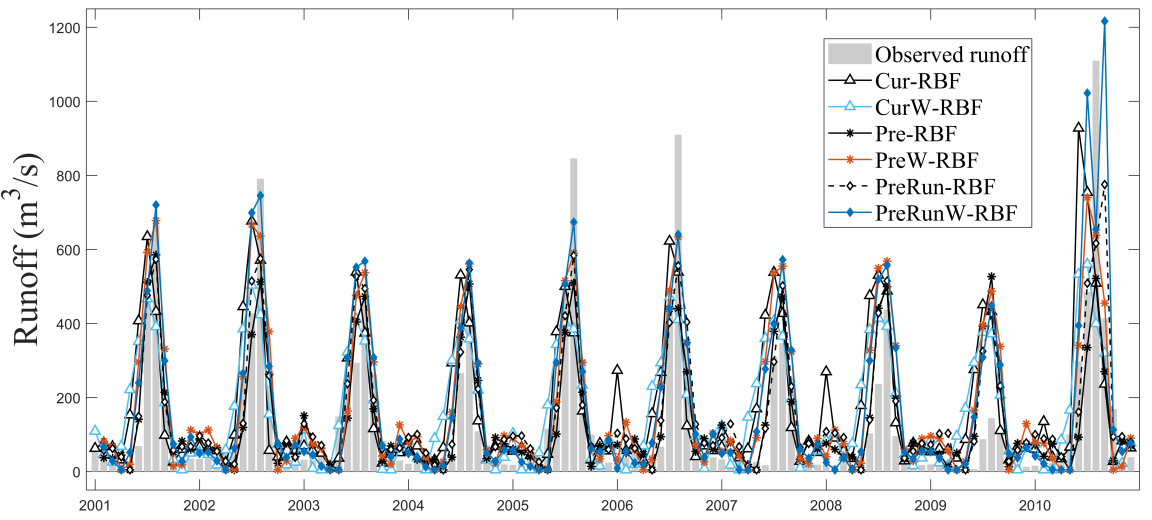

(b) 


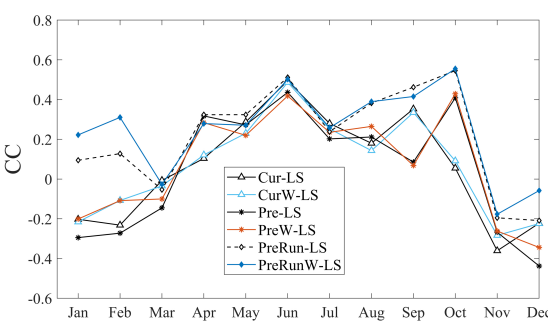

(a)

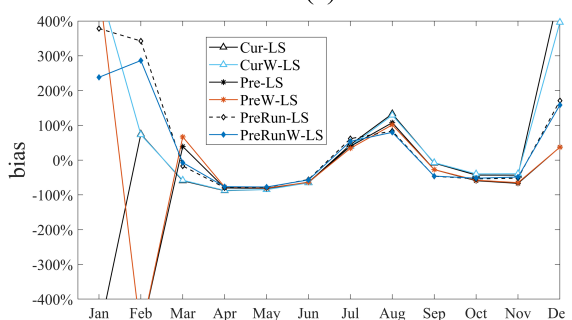

(c)

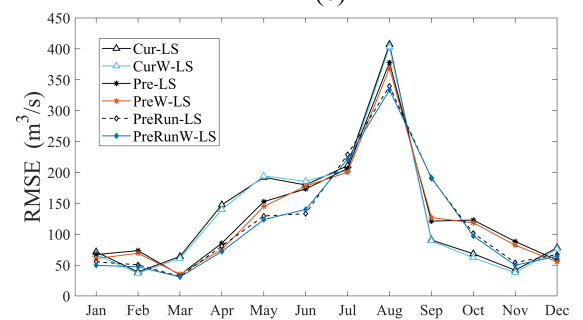

(e)

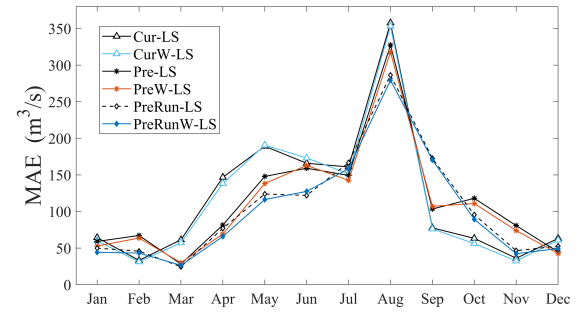

(g)

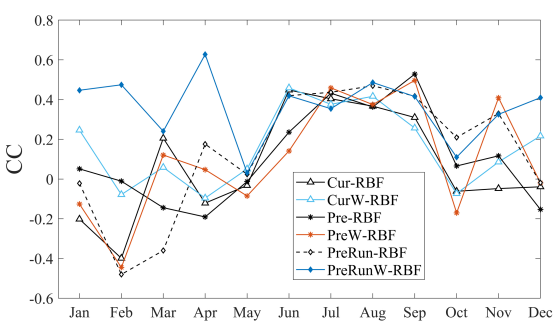

(b)

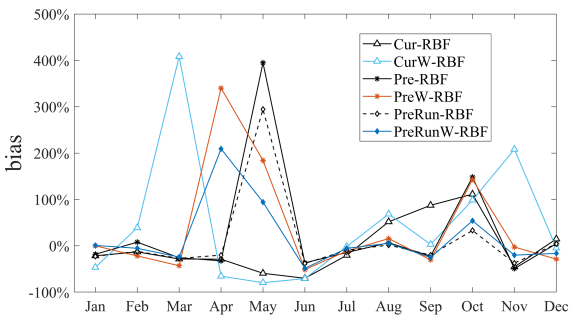

(d)

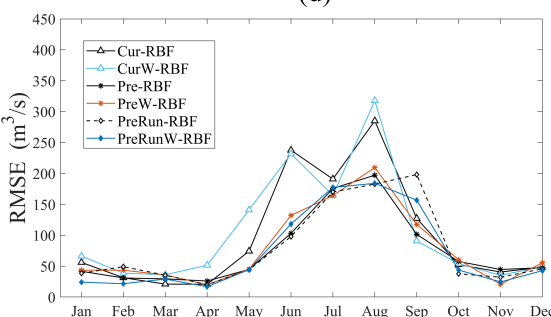

(f)

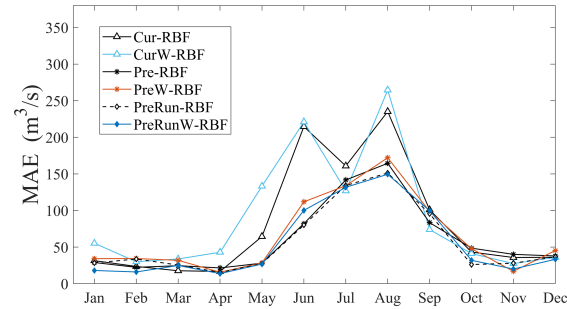

(h) 
Table 1. Change rates of monthly mean precipitation, temperature and runoff in the upper Tarim River basin during the period 1961-2010.

\begin{tabular}{|c|c|c|c|c|c|c|}
\hline Trend & Jan & Feb & Mar & Apr & May & Jun \\
\hline $\begin{array}{c}\text { Precipitation } \\
\text { trend }(m m / \text { month })\end{array}$ & 0.052 & 0.042 & 0.063 & 0.019 & 0.054 & 0.158 \\
\hline $\begin{array}{c}\text { Temperature } \\
\text { trend }\left({ }^{\circ} \mathrm{C} / \text { month }\right)\end{array}$ & 0.026 & 0.044 & 0.029 & 0.018 & 0.017 & 0.012 \\
\hline $\begin{array}{c}\text { Runoff trend } \\
\left(\mathbf{m}^{3} /(\mathbf{s} \cdot \text { month })\right)\end{array}$ & -1.125 & -0.950 & -0.324 & 0.352 & 1.008 & -0.023 \\
\hline Trend & Jul & Aug & Sept & Oct & Nov & Dec \\
\hline $\begin{array}{c}\text { Precipitation } \\
\text { trend(mm/month) }\end{array}$ & 0.124 & 0.033 & 0.137 & 0.087 & 0.036 & 0.046 \\
\hline $\begin{array}{c}\text { Temperature } \\
\text { trend }\left({ }^{\circ} \mathrm{C} / \text { month }\right)\end{array}$ & 0.006 & 0.016 & 0.017 & 0.019 & 0.047 & 0.045 \\
\hline $\begin{array}{l}\text { Runoff trend } \\
\left(\mathbf{m}^{3} /(\mathbf{s} \cdot \text { month })\right)\end{array}$ & -1.806 & -1.515 & 0.212 & 0.115 & -1.222 & -1.801 \\
\hline
\end{tabular}


Table 2. Summary of models based on different independent variables

\begin{tabular}{cccccccc}
\hline & \multicolumn{1}{c}{ Current } & \multicolumn{3}{c}{ Preceding } & Linear fitting & Non-linear fitting \\
Model & $\mathrm{P}^{\text {cur }}$ & $\mathrm{T}^{\text {cur }}$ & $\mathrm{P}^{\text {pre }}$ & $\mathrm{T}^{\text {pre }}$ & $\mathrm{R}^{\text {pre }}$ & Least-Squares & Radial-Basis Function \\
\hline Cur & $\sqrt{ }$ & $\sqrt{ }$ & - & - & - & Cur-LS & Cur-RBF \\
CurW & $\sqrt{*}$ & $\sqrt{*}$ & - & - & - & CurW-LS & CurW-RBF \\
Pre & $\sqrt{ }$ & $\sqrt{ }$ & $\sqrt{ }$ & $\sqrt{ }$ & - & Pre-LS & Pre-RBF \\
PreW & $\sqrt{*}$ & $\sqrt{*}$ & $\sqrt{*}$ & $\sqrt{ } *$ & - & PreW-LS & PreW-RBF \\
PreRun & $\sqrt{ }$ & $\sqrt{ }$ & $\sqrt{ }$ & $\sqrt{ }$ & $\sqrt{ }$ & PreRun-LS & PreRun-RBF \\
PreRunW & $\sqrt{*}$ & $\sqrt{*}$ & $\sqrt{*}$ & $\sqrt{*}$ & $\sqrt{ }$ & PreRunW-LS & PreRunW-RBF \\
\hline
\end{tabular}


Table 3. Summaries of the statistical results based on different models, against the runoff observations, in the upper Tarim River basin during the period 2001-2010.

\begin{tabular}{lllll}
\hline Model & CC & Bias $(\%)$ & RMSE $\left(\mathrm{m}^{3} / \mathrm{s}\right)$ & MAE $\left(\mathrm{m}^{3} / \mathrm{s}\right)$ \\
\hline Cur-LS & 0.59 & 14.91 & 163.85 & 107.76 \\
CurW-LS & 0.62 & 15.43 & 160.05 & 103.01 \\
Pre-LS & 0.69 & 22.90 & 151.34 & 104.63 \\
PreW-LS & 0.72 & 20.50 & 143.61 & 98.05 \\
PreRun-LS & 0.73 & 19.22 & 141.45 & 95.32 \\
PreRunW-LS & 0.75 & 17.15 & 136.40 & 89.33 \\
Cur-RBF & 0.69 & 28.17 & 161.17 & 104.60 \\
CurW-RBF & 0.71 & 15.95 & 143.31 & 90.41 \\
Pre-RBF & 0.85 & 33.68 & 126.08 & 84.38 \\
PreW-RBF & 0.84 & 29.61 & 120.86 & 82.90 \\
PreRun-RBF & 0.82 & 41.06 & 146.75 & 87.95 \\
PreRunW-RBF & 0.83 & 30.19 & 136.20 & 75.42 \\
\hline
\end{tabular}

